\title{
Presentación. Alquimia de la tierra
}

Presentation. Alchemy of the soil

\section{Elena Freire Paz}

Departamento de Filosofía e Antropoloxía, Universidade de Santiago de Compostela (España) elena.freire@usc.es

\author{
ALFARERÍA POPULAR, LA MEMORIA EN LAS MANOS \\ MONOGRÁFICO COORDINADO POR ELENA FREIRE PAZ (Universidad de Santiago de Compostela)
}

\begin{abstract}
RESUMEN
El presente monográfico tiene por objetivo realizar una lectura de la alfarería popular en perspectiva antropológica, para lo que resultan fundamentales las claves socioeconómicas y socioculturales dentro de las cuales se desarrolla el oficio y, por tanto, lejos de cualquier percepción esencialista. Así, los diversos análisis que lo integran afrontan la pervivencia del oficio en lugares muy dispersos geográficamente (Timor, El Rif, México, Costa Rica y España) y bajo circunstancias muy dispares, que oscilan desde el olvido y la marginalidad hasta su uso político como símbolo de identidad, sin olvidar en la mayoría de los casos su aprovechamiento como recurso económico vinculado al turismo. Observar las razones y los contextos por los que, en pleno siglo $\mathrm{XXI}$, distintos entramados culturales mantienen elementos del pasado amparados por su catalogación artesanal consideramos que mejora el estudio de una realidad que responde de forma local a la globalización en función de dinámicas de máximo interés para ser analizadas por las ciencias sociales de forma rigurosa más allá de su clasificación genérica, igualitaria y meramente descriptiva en la categoría folclórico.
\end{abstract}

\section{ABSTRACT}

The objective of this monograph is to carry out a reading of popular pottery from an anthropological perspective. For this, the socio-economic and socio-cultural keys within which the trade is developed are fundamental and, therefore, far from any essentialist perception.Then, the analysis that comprise it face the survival of the trade in geographically widely dispersed places (Timor, El Rif, Mexico, Costa Rica and Spain) and under very disparate circumstances, ranging from oblivion and marginality to its political use as a symbol identity, without forgetting in most cases its use as an economic resource linked to tourism. Observing the reasons and the contexts by which, in the XXI century, different cultural frameworks maintain elements of the past protected by their artisan cataloging, we consider that it improves the study of a reality that responds locally to globalization based on dynamics of maximum interest to be analyzed by the social sciences in a rigorous way beyond its generic, egalitarian and merely descriptive classification in the folkloric category.

PALABRAS CLAVE

alfarería popular | artesanía | identidad | género femenino | patrimonio

KEYWORDS

pottery | handicraft | identity | female gender | heritage

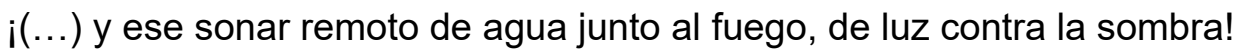 (Octavio Paz, "El cántaro roto")}

\begin{abstract}
En 1985 Claude Levi-Strauss publicaba en París una de sus obras fundamentales, La potière jalouse, en la que se recogían las líneas generales de sus aportaciones teóricas a la antropología, combinadas con los datos de campo obtenidos en sus investigaciones sobre la cultura americana. Desde ese mismo título, la artesanía hecha a partir de barro pasó a ocupar un lugar central como catalizador para una nueva consideración antropológica del ser humano. Según el autor francés, en la alfarería se representa un combate simbólico entre dos mundos, uno celestial y otro terrenal, donde la intermediación del fuego termina por ensamblar la lógica de los mitos al tiempo que construye la amalgama perfecta de todos los componentes que constituyen cada pieza elaborada con arcilla. Sería, precisamente, sobre ese pensamiento mítico donde descansaría cualquier pregunta que el ser humano pudiese realizar a propósito del significado. Para comprender la compleja significación de la alfarería no se puede perder de vista que en ella están presentes los cuatro elementos clásicos (tierra, agua, aire y fuego) que constituyen el fundamento material de lo existente y cuya fusión significa, de hecho, la primera formulación de la alquimia de tal forma que el más simple de los objetos realizados con barro encierra en su interior la total complejidad del pensamiento.
\end{abstract}


Es posible que en esa publicación se encuentre una de las reflexiones más profundas y de mayor alcance alrededor de un mundo, el de la alfarería, presente en multitud de trabajos que engrosan, aunque solo fuese por acumulación, un corpus muy significativo para quienes puedan estar interesados en el estudio de esta artesanía. Desde luego, resulta innegable la potencialidad evocadora del barro para los seres humanos a través de las diferentes culturas, hasta el punto de que ha llegado a colocar a quienes dominan las técnicas de elaboración en un estrato simbólico que es propio de la deidad creadora. En este sentido son muy variadas las culturas en las que la creación de hombre por parte de una entidad superior se ejecuta a través de la manipulación y cocción del barro o bien, en algunas otras variantes, asociada al acto de cocinar, en el que resulta muy sencillo identificar las mismas fases que en el ejercicio de la alfarería. Al mismo tiempo, la alfarería, en cualquiera de sus usos, ya sean cotidianos - vinculados, precisamente, con la comida - ya rituales, concentra multitud de significaciones de máxima complejidad como, por ejemplo, entre otras, la dicotomía naturaleza/cultura.

Con independencia de todas estas controversias cabría preguntarse por las razones para dedicar, a estas alturas del siglo XXI, un monográfico en una revista de reconocida trayectoria, como es Gazeta de Antropología, a la alfarería tradicional. A nuestro juicio, la respuesta debería de ser al menos doble.

En primer lugar, por la indudable cotización de la artesanía del barro como elemento de significación, ya que la alfarería representa un universo simbólico inagotable en función de su propio valor como condensador cultural, una circunstancia solo compartida, posiblemente, dentro de las muchas actividades artesanales que han acompañado a los seres humanos desde la antigüedad, con otro oficio, la tejeduría.

Por otra parte, desde una perspectiva mucho más pragmática, el interés por la cerámica de basto en la actualidad estaría más que justificado si atendemos a su pervivencia en muy variados espacios geográficos, sociales y culturales y en un estadio sincrónico que encierra dentro de sí otros tiempos múltiples. Así enfocada, la alfarería tradicional se constituye como un campo de trabajo favorable para las Ciencias Sociales en general y para la Antropología Social y Cultural en particular, en tanto que su análisis nos permite observar los procesos socioeconómicos y culturales que la acompañan. ¿Por qué, cómo, dónde y para qué los seres humanos siguen haciendo barro a estas alturas de la historia? ¿Cuáles han sido los cambios y las continuidades de un oficio vinculado de forma atemporal y perenne con el mito, bajo el manto siempre protector de la tradición, pero vertebrado, producido y consumido en un tiempo presente?

En un ejercicio de confluencia analítica de ambos factores, su combinación acreditaría, con solvencia, la seducción de los objetos realizados con barro en la sociedad actual como contenedores culturales de primer orden y, por lo tanto, justificaría la aparición de este volumen. $\mathrm{Y}$, sin embargo, existe aún otra razón que explica, además, la dispersión, en términos geográficos, y no solo, de los lugares escogidos para representar la alfarería tradicional en esta publicación a los cuales nos dedicaremos con detalle más adelante. Nos estamos refiriendo al tratamiento que los productos elaborados con arcilla han tenido desde la disciplina antropológica, que ha dejado importantes espacios tendentes a la carencia de análisis e interpretación. Resulta muy interesante observar, por ejemplo, en un repaso bibliográfico, aunque sea somero, sobre el barro en España, cómo este está presente en numerosas publicaciones desde hace décadas - como integrante del folklore el lapsus temporal tendría que ser notablemente ampliado-, pero, de manera generalizada, manteniéndose en un relato meramente descriptivo de las piezas y, a lo sumo, del instrumental técnico requerido para su realización. Como decíamos, no puede dejar de resultar muy llamativo que la simple presencia de los objetos de alfarería parezca resultar suficiente, incontestable y absoluta hasta el punto de que hace prescindible, cuando no anula, la existencia de análisis, reflexión y argumentación en torno a los grupos que los generan o a los contextos en los que se producen. En este sentido, tal pareciese que aquello que ha conformado a las piezas de barro como dignas merecedoras de atención, vinculándolas a las obras de arte, las ha alejado, por completo, de la cotidianidad en la que se producen y cobran sentido. Ha sido esta particularidad, en la que la atención prestada se focaliza sobre los objetos, terminando por hacer desaparecer todo el soporte cultural que los crea, la que ha terminado por situar el tratamiento que se ha otorgado a la alfarería tradicional dentro del conocimiento antropológico en una especie de dibujo fuera del margen a modo de "cronopio". Pues bien, a nuestro entender, explorar esa forma de otredad, lejos de resultar limitante supone un incentivo de máximo interés para la investigación. He aquí la tercera, y ahora sí última, de las razones por las que nos hemos decidido a elaborar el presente dossier.

Nos referíamos, unas líneas más arriba, a la heterogeneidad de las alfarerías que están presentes en este volumen en el que los lectores tendrán la oportunidad de aproximarse a las coordenadas en las que 
las labores del barro tradicionales perviven en contextos muy diversos a nivel mundial. Quienes se puedan sentir interesados en la lectura atenta y/o la consulta de este número podrán conocer y profundizar en aspectos de la artesanía del barro realizada en localizaciones como Timor Leste, Cabo Verde, el Rif, Brasil, Costa Rica y México, para terminar con un estudio de caso ubicado en España. Planteado en movimiento de espiral galaica, en realidad, ese viaje de ida lo es también de vuelta y el equilibrio del monográfico viene dado por la posibilidad que ofrece de mostrar, en modo panorámico, un recorrido por distintos transcursos de la actividad alfarera en Asía, África, América y, finalmente, Europa, que se encuentran dentro de un mundo que parece hiperconectado, pero que se manifiesta altamente diferenciado en las prácticas locales. He ahí una de las aportaciones más preciadas del volumen: poder llevar a cabo una comparación de la diferencia desde la uniformización de un saber-hacer compartido.

El orden de los artículos responde al interés por un relato que explore la otredad como distancia de la identidad propia. Así, a modo de apertura, nos hemos decantado por la reflexión propuesta por Paulo Seixas sobre la posibilidad de que el barro haya funcionado, para distintas sociedades de tradición oral, como una forma de registro material no escrito y, por lo tanto, como soporte comunicacional que opera a distintos niveles (producción, consumo, observación/contemplación) y que permite asumirlo como un ejercicio de auto-representación.

En su texto, María José Matos y Jorge Wagner plantean una interesante y detallada recopilación de datos en la que se hace evidente el arcaísmo de la cerámica producida por distintas cabilas o tribus asentadas en las dos laderas de la cordillera del Rif. La importancia de la cerámica rifeña viene dada, en lo que respecta a nuestra publicación, porque resulta un ejemplo claro de agónica pervivencia sin intervención y, por tanto, pone en cuestión el hecho de que la artesanía del barro sea susceptible de ser conservada por su alto valor patrimonial entendiendo este de forma esencialista. El Rif es la prueba de que allí donde no hay intereses económicos y/o políticos no se construye patrimonio con independencia de la existencia de artefactos culturales susceptibles de ser identificados como una especie de cápsulas del tiempo con las que sería posible construir un relato de permanencia cuasi atemporal que debería funcionar como aval máximo para captar algún interés que permitiese su catalogación y conservación.

Precisamente esa identificación de lo artesanal como algo auténtico ha sido una de las claves que ha permitido el mantenimiento de la alfarería tradicional en diferentes localizaciones de México. Unas producciones cerámicas en las que resulta obvio encontrar el punto de unión con la alfarería del Rif en el hecho de que todas ellas eran producidas por mujeres y, sin embargo, ha sido la introducción de claves foráneas, como pueden ser el fomento de cooperativas auspiciadas desde diferentes instituciones estatales e internacionales o la demanda turística, la que ha dado lugar a cambios significativos en las producciones locales. Estos son los casos de la alfarería tradicional producida en Metepec con el trabajo firmado por Guadalupe Núñez Núñez, Graciela Cruz Jiménez y Rocío del Carmen Serrano Barquín, y la alfarería de Capula (Michoacán) analizada por Amalia Ramírez Garayzar. En ellos es posible observar esa construcción del patrimonio que no existía en la alfarería del Rif y que busca acomodarse al presente bajo una narrativa que evoca lo perenne pero que se origina mientras se da cabida a recreaciones identitarias que solo resultan explicables a través de un proceso, como mínimo, de hibridación, que potencia la invención de la tradición y cuyo caso paradigmático estaría constituido por la elaboración de las catrinas.

Esas mismas claves de pervivencia a cambio del acceso a los circuitos turísticos y, por lo tanto, arrastrados por el flujo económico bajo la etiqueta de lo auténtico con el amparo/intervención institucional, también son las que están operando en el caso de la alfarería producida en Nicoya (Costa Rica) que han estudiado Anayensy Herrera y Jim Weil. Aquí llaman la atención, al menos, dos peculiaridades muy significativas.

En primer lugar, el hecho de que en una artesanía elaborada tradicionalmente por las mujeres los hombres se hayan incorporado masivamente en el momento en el que se ha producido una capacitación técnica en torno al oficio (justo lo opuesto a lo ocurrido en el último ejemplo de caso que conforma el monográfico y que veremos a continuación).

En segundo lugar, vinculado con esa invención de la tradición a la que nos referíamos, la apertura de una línea de producción específica para la reproducción de lo auténtico, que en este caso se identifica con piezas arqueológicas que han pasado a ser producidas por la artesanía local para satisfacer la demanda turística que expresa un evidente gusto por lo precolombino. En este sentido se manifiesta, de nuevo, la presentación como propio de lo que, en realidad, resulta filtrado a través las expectativas de los forasteros que acuden a esos lugares para buscar lo que, de alguna manera, ya han encontrado. 
El último de los trabajos, firmado por Freire Paz, aborda un estudio de caso en el que los factores ideológicos y políticos han supuesto un añadido nada desdeñable sobre los factores de carácter económico para entender las razones que motivaron la elección de la alfarería tradicional como metáfora de un determinado modelo de reivindicación nacional. Así, el monográfico se cierra con un trabajo que presenta una comunidad autónoma española (Galicia) que ha erigido su identidad sobre una tradición intencionalmente contenida en recipientes de barro, y ha sido esta circunstancia, precisamente, la que ha motivado la permanencia del oficio en unas coordenadas diferenciadas con respecto al conjunto del estado español.

No podemos evitar realizar una especial mención sobre uno de los hilos invisibles con los que hemos tejido el presente volumen y que, aún sin constituir una aproximación a la alfarería tradicional desde la perspectiva de género, sí permite prestar una adecuada atención al tránsito de las féminas por el oficio. En este sentido, queremos destacar el particular interés las menciones a lo femenino en la totalidad de los textos que conforman la monografía ya que, bien sea en forma de datos, de simples apuntes o de especial reflexión, da cuenta de la variabilidad de las circunstancias en las que las mujeres participan y/o han participado de esa actividad. Esta volatilidad, en función de los contextos y de los momentos, sobre los roles desempeñados por las mujeres en torno a la cerámica de basto constituye un indicador de primer orden para percibir la importancia de aportar etnografías que describan las distintas prácticas posibles sobre un mundo asociado a la inmutabilidad, a la permanencia y a la inmanencia que semejan desprender las piezas de barro. Existe, aún, otra cualidad que ensambla la cacharrería con lo femenino y es la identificación de ambos universos con lo subalterno y con el anonimato lo que no deja de constituir una forma de marginalidad y de silencio dentro de la conformación del entramado social. Así, frente al arte y al androcentrismo estarían las artesanías y las mujeres tejiendo la urdimbre de lo cotidiano que mantiene la vida.

Debemos agradecer a los autores el esfuerzo por participar en una publicación que trae a la primera línea del debate científico el mundo del barro, intentando eliminar esas barreras expositivas de las que ya hemos hablado y que lo elevan en la misma medida en la que, probablemente, lo han constreñido. Poner el foco sobre algo más que las piezas, sin que ello suponga, en ningún caso, restar a estas ni lo más mínimo de su valor, sino todo lo contrario, ha sido un objetivo común a todas las personas que han colaborado con sus textos, de la misma forma que lo ha sido visibilizar la importancia que tiene su pervivencia en los diferentes contextos.

En nuestros agradecimientos tenemos también que considerar el soporte económico ofrecido por el grupo de investigación Gl1428-Antropoloxía socio-cultural que dirige la profesora Nieves Herrero Pérez, así como el de la Facultade de Humanidades y el Vicerreitorado de Coordinación del Campus de Lugo de la Universidade de Santiago de Compostela, que han contribuido también a que otras miradas sobre la artesanía del barro de basto hayan podido conformar este compendio de trabajos en perspectiva antropológica, que esperamos resulte de interesante lectura, y como llamada de atención sobre el tratamiento que los grupos humanos hacemos de determinados elementos de la cultura. 